\title{
LETTER Special Section on Empirical Software Engineering \\ Empirical Study on Improvements to Software Engineering Competences Using FLOSS
}

\author{
Neunghoe $\mathrm{KIM}^{\dagger \mathrm{a})}$, Member, Jongwook JEONG ${ }^{\dagger, \dagger \mathrm{b})}$, and Mansoo HWANG ${ }^{\dagger \dagger \dagger \mathrm{c})}$, Nonmembers
}

SUMMARY Free/libre open source software (FLOSS) are being rapidly employed in several companies and organizations, because it can be modified and used for free. Hence, the use of FLOSS could contribute to its originally intended benefits and to the competence of its users. In this study, we analyzed the effect of using FLOSS on related competences. We investigated the change in the competences through an empirical study before and after the use of FLOSS among project participants. Consequently, it was confirmed that the competences of the participants improved after utilizing FLOSS.

key words: FLOSS, software engineering competence, requirement management tool, software education, user competence analysis

\section{Introduction}

Most free/libre open source software (FLOSS) can be modified and used for free [1]; therefore, many companies and organizations are increasingly using the appropriate FLOSS for their desired purpose. Furthermore, companies utilize FLOSS projects to acquire user innovations, reduce costs, and earn money through complementary services [2].

In the field of software education, recent studies [3] indicated that the skill of software developers improved using tools; therefore, we assumed that the usage of FLOSS in a software development project will improve related competences. In this study, we empirically explored the changes in participants' competences due to FLOSS in software development for requirement management. We measured the competences of the project participants before and after using FLOSS; consequently, the effect of using FLOSS on the competences of the participants was analyzed.

\section{Empirical Study Design}

\subsection{Setting of the Case Study}

A database specialized software company in Korea that develops and sells its own software package was selected

Manuscript received February 28, 2019.

Manuscript revised July 1, 2019.

Manuscript publicized September 24, 2019.

${ }^{\dagger}$ The authors are with Department of Computer, Korea University, Seoul, Korea.

${ }^{\dagger}$ The author is with Department of Computer Science, Korea Military Academy, Seoul, Korea.

${ }^{\dagger \dagger}$ The author is with School of IT Convergence Engineering, Shinhan University, Dongducheon, Korea.

a)E-mail: nunghoi@ korea.ac.kr

b) E-mail: jwjeong55@gmail.com

c) E-mail: mshwang@ @ shinhan.ac.kr (Corresponding author) DOI: 10.1587/transinf.2019MPL0001 as the case study; half of their packages are sold to Japan, China, and Taiwan. The key services of the company include database management, access control, encryption, vulnerability analysis, and other services required for databases.

The project that we investigate develops a solution to protect information from threats by recording blocking and access history using DB access control. This project faced problems other than the development tasks. Owing to the complicated usage environment, customers frequently requested customization; however, because of an inefficient management system, redundant development efforts occurred. This project includes several activities that are closely related to the requirement, which is appropriate considering the objective of our investigation.

For this study, five competences were measured. A company choses eight participants closely related to requirement management in the project, among whom five qualified participants were selected for the case study.

\subsection{Application of FLOSS in Case the Case Study}

The company decided to use FLOSS because they did not have a specialized requirement management tool. At that time, the requirements were handled independently by each team; that is, they were not shared or managed across different teams in the organization.

For this project, they selected an open source requirement management tool with a several downloads that were provided by a government agency of Korea. The tool supports requirement elicitation, analysis, and specification and change management for requirement management.

\subsection{Procedure}

The requirement engineering competences of the project participants and environment of the company was investigated before applying FLOSS to the project. In particular, the requirement engineering competence status was examined in four areas: requirement elicitation, analysis, specification, and change management. In this step, a software consulting group attended, comprising five participants who had more than 3 years of experience in various developmental areas. All 29 items of the questionnaire were individually rated on a 5-Likert scale. Before the initiation of project development, we conducted a pre-survey. At the end of project, a post-survey was conducted. 


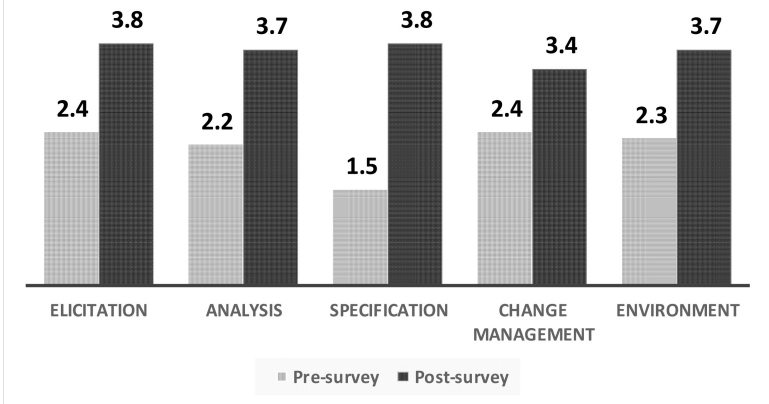

Fig. 1 Average of measured participants' competences in surveys.

\section{Results and Discussions}

\subsection{Measured Participants' Competences}

The average pre-survey results are as follows: 2.4 points in requirement elicitation, 2.2 points in requirement analysis, 1.5 points in requirement specification, 2.4 points in requirement change management, and 2.3 points in the requirement engineering environment. The total measured points were 10.8 points from a total of 25 points. The overall score was not high, and the requirement specification area showed the lowest score.

The average post-survey results are as follows: 3.8 points in requirement elicitation, 3.7 points in requirement analysis, 3.8 points in requirement specification, 3.4 points in requirement change management, and 3.7 points in the requirement engineering environment. The total measured points were 18.4 points from a total of 25 points. In all respects, the score was increased with an average increase of 1.52, and the requirement specification area showed the highest improvement.

We confirmed that measured participants' competences were normally distributed using the Shapiro-Wilk test (significance level $=0.05$ ). Using the $t$-test (significance level $=$ 0.05 ), the $p$-value was determined as 0.002 and we confirmed that the difference is statistically significant. As a result, average of the post-survey $(3.68 \pm 0.16)$ is higher than that of the pre-survey $(2.16 \pm 0.37)$.

\subsection{Analysis and Discussions}

The benefits FLOSS provided to companies are not discussed because it is outside the scope of this study, the
FLOSS used in the project provided appropriate benefits as a tool for requirements collaboration and sharing. Furthermore, while using FLOSS, we confirmed that participants' competences related with requirement engineering were improved unintentionally. This shows that, although FLOSS does not explicitly provide education services, it helps improve participants' competences and environments in related fields, which was improved performance particularly because the investigated company heavily relied on an individual's competences. Based on these results, requirements management are expected to be performed at a high level owing to the improvement of the participants' competences. Moreover, software companies and organizations invest significant time and cost to improve their employees' software engineering competences; FLOSS helps achieve this goal without requiring additional time and cost.

\section{Conclusion}

We empirically investigated and analyzed the changes in competences of project participants due to FLOSS for requirement management. Consequently, improvements in the related competences of the participants were observed in addition to the intended benefits. This study revealed that the usage of FLOSS can benefit not only the functional competences but also the software engineering competences of participants.

\section{Acknowledgments}

This research was supported by Basic Science Research Program through the National Research Foundation of Korea (NRF) funded by the Ministry of Education (2018R1A6A3A01013334).

\section{References}

[1] Z. Abdellah, A. AmineArrahmane, and J. Fatima, "Factors affecting adoption of FLOSS ERP system by SMEs' directors and managers in developing countries using UTAUT2, SEM and R," Proc. 4th IEEE International Colloquium on Information Science and Technology, Tangier, Morocco, pp.25-30, Oct. 2016.

[2] Y. Zhang, X. Tan, M. Zhou, and Z. Jin, "Companies' domination in FLOSS development: an empirical study of OpenStack," Proc. 40th International Conference on Software Engineering: Companion Proceedings, Gothenburg, Sweden, pp.440-441, May 2018.

[3] A. Aloulou and O. Boubaker, "Enhancing technical skills of control engineering students in robotics by using common software tools and developping experimental platforms," Proc. Intl. Conf. on Education and e-Learning Innovations, Sousse, Tunisia, pp.1-5, July 2012. 